\title{
The pledge rate research of copper and zinc inventory portfolio based on Copula-VaR method
}

\author{
Li Zhou ${ }^{1, ~ *, ~ J i n g ~ D o n g ~}{ }^{2}$ \\ ${ }^{1}$ School of Information, Beijing Wuzi University, Beijing, China \\ ${ }^{2}$ Graduate department, Beijing Wuzi University, Beijing, China \\ Email address: \\ zhoulibit@126.com (Li Zhou)
}

To cite this article:

Li Zhou, Jing Dong. The Pledge Rate Research of Copper and Zinc Inventory Portfolio Based on Copula-VaR Method. Journal of Investment and Management. Vol. 3, No. 2, 2014, pp. 37-41. doi: 10.11648/j.jim.20140302.11

\begin{abstract}
This paper selects copper and zinc as a stock portfolio of mortgage financing. It calculates stock portfolio return risk by the value at risk method, introduces Copula function to measure the two pledges' relationship, simulates portfolio yield trend by Monte Carlo method to get Var value. At last compare Copula-Var method with traditional method, The new method can reflect more collateral prices spike characteristics, estimated loss better when value at risk. The conclusion of the study establishes the foundation for further study on the theory and methods of inventory financing portfolio.
\end{abstract}

Keywords: Copula, VaR, Inventory Portfolio, Pledge Rate

\section{Introduction}

The pledge rate as an important tool for assessment and management of risk of inventory pledge, gradually attention by researchers, and some scholars also use model analysis and research on it. Pledge Loans origin from overseas, pledge rate research is earlier in foreign country, while the research on pledge rate of China is still at the starting stage.

Jokivuolle and Peura (2003) [1] proposes a model about the relationship between the pledge value and default risk probability, and then use the model to study the expectations default loss. Reasonable loan ratios are their research contents. Cossin and Hricko (2003)[2] analyzes credit risk by using the structural method, focuses on the mortgage credit risk, points out that in different circumstances, collateral impact on credit risk is not the same, cannot use a simple model to judge, separately lists double default collateral, random collateral, random interest rates under the random mortgage bonds, continuous and discrete mark to market margin recovery etc..

The domestic scholars, Li Yixue (2007) [3] in the basic model on the loan value ratios, assumption of corporate default as exogenous variables and follows a stochastic Poisson process, adjusts the model. Qi Ershi (2008) [4] study combination loan pledge rate, using the Copula function to describe the price of commodity collateral, and assume that commodity price movements are normal Copula function, uses the method of credit risk structure model building the probability function of corporate default, finally solves pledge rate by nonlinear programming. Gao Jie (2009) [5] discussed in dynamic logistics supervision mode, enterprise credit level and the collateral price effects pledge rate, is established calculation model for the batches repeatedly inventory pledge rate. Sun Zhaoyuan (2011) [6] studied the pledge rate of double category stock portfolio, in static pledge mode, assumes that the price change of inventory pledge portfolio obeys independent lognormal distribution, and then constructs the pledge rate model.

In practice, enterprises maybe have more than one kind inventory in pledge financing, maybe use a variety of inventory financing, a variety of collateral may be raw materials, semi-finished or finished products, while the price changes between these pledge is related, such as the rising prices of raw materials, finished goods price will go up, when raw material prices fall, product prices will fall. If failure to consider the correlation of these pledge of price changes in inventory financing, it implied the potential business risks.

\section{The Copula-VaR Method is Applied to the Stock Portfolio Mortgage Rate}

\subsection{Value at Risk}

The bank believes that "value at risk" is more important than the "risk", which laid the foundation for the development of VaR. 
At the same time, people more concerns about the risk of proper management of financial derivatives problem, JP Morgan as the member of group of 30 (G30), provides a communication platform for the industry to discuss best practices for risk management. In 1993 July, a report issued by the group of 30 nations, pioneered use the term of value at risk. With the wide spread of the report, the term spread.

\subsection{Defination of VaR}

Philippe Jorion defines $\mathrm{VaR}$ as in normal market conditions, given certain confidence level, expected future losses within a period of time. Defined $c$ as the confidence level, $\mathrm{X}$ for portfolio loss, VaR says absolute value of the holding period within the $T$ the loss of definition, its formula is

$$
\operatorname{Pr} o b(X \leq V a R)=c \text { or } \operatorname{Pr} o b(X \geq V a R)=1-c .
$$

VaR value is the confidence level, the maximum loss of the portfolio at future $\mathrm{T}$ period. For example a more vivid understanding, assuming a fund managers hope that within the next 10 days, with $95 \%$ probability that the management of the fund's losses of less than $\$ 1000000$, then it can be written as:

$$
\operatorname{Pr} o b(X \leq 1000000)=95 \% \text { or } \operatorname{Pr} o b(X \geq 1000000)=5 \%
$$

\subsection{The General Calculation Formula of VaR}

According to the definition of VaR, when the value of assets in the period known, $X$ is equal to the difference between maximum value and the first value loss, VaR can be calculated by the formula. But in reality, it is difficult to obtain the specific value $X$, it is assumed that the return on assets approximately obey a certain distribution to predict the assets, in the calculation of the value change of return rate during period to calculate VaR.

Definition $P_{t}$ is price in time $\mathrm{t}, t=1,2, \cdots, T$, then, return rate $R_{t}$ is $R_{t}=\ln P_{t}-\ln P_{t-1}$, the price of assets: in time $t+1$ is $P_{t+1}=P_{t} \times \exp \left(R_{t+1}\right)$, we can know, when yields at minimum value the assets value is the minimum, easy to obtain VaR.

Let $\mathrm{R}$ be the random variable of asset returns, $f(x)$ is the probability density function, $R^{\min }$ is the minimum rate of return, under the given confidence level of $c$,

$$
\operatorname{Pr} o b\left(R>R^{\min }\right)=\int_{R^{\min }}^{+\infty} f(x) d x=c .
$$

\subsection{The Calculation Method of VaR}

So far, there are three main methods to measure the VaR, Delta-normal distribution method, historical simulation method and Monte Carlo method.

\subsubsection{Delta-normal Distribution Method}

Assuming that the rate of investment return follows a normal distribution, the normal distribution has a characteristic that the confidence and quintile corresponding risk, value can be calculated using a combination of the characteristics of the yield, then the risk value is equal to the investment portfolio with established confidence quintiles corresponding product level:

$$
V a R=W_{0} \times z_{\partial} \times \sigma \times \sqrt{\Delta t}
$$

Where, $W_{0}$ is the initial investment is established, $z_{\partial}$ is $\partial$-quantile under the corresponding confidence level of $\partial, \sigma$ is the standard deviation of portfolio return rate, $\Delta t$ as the investment period.

According to the above formula can be seen, the calculation of VaR according to the two important parameters is the confidence level and duration of investment, risk manager and investment object is different, these two parameters will be different. The disadvantage of this method is the rate of return on assets obeys the normal distribution assumption, ignoring asset return possible peak thick tail characteristics.

\subsubsection{Historical Simulation Method}

The historical simulation method based on the historical data to simulate the portfolio future rate of return distribution, similar with the delta-normal method, at a confidence level are given, according to percentile calculation corresponding to VaR. The key of the simulation is that the future of portfolio weights and history weights is consistent.

The historical simulation method is better understanding from the concept, the calculation is relatively simple, do not like the delta-normal method for distribution assumption, and, the historical simulation method can also deal with non-normal distribution, non-symmetrical distribution of the thick tail, under certain conditions, can also for the nonlinear problem of processing. But the disadvantage is that assumes that risk factors and historical data in the market completely, in the use of historical simulation method, need a large amount of historical data, when the data are not good collection, historical simulation method is very easy to have the risk.

\subsubsection{Monte Carlo Method}

Monte Carlo method and the historical simulation method is like, are required to carry out the simulation calculation. Monte Carlo data change is not according to the historical data, but through random simulation obtained. The basic thought of Monte Carlo method is the asset return rate obeys a random distribution, utilizes the computer to simulate, generate random data of assets rate of return during holding period, then calculate VaR according to the random data obtained.

Monte Carlo method mainly includes the following three steps:

Step 1, choose the appropriate random process, and describe the prices of assets.

Step 2, simulate asset price in accordance with the 
selected random process.

Step 3, on the basis of the simulation results, construct the distribution of asset returns, and calculate portfolio risk.

Monte Carlo method has the advantages that the risk of price fluctuation can cover assets, also can deal with non-normal distribution, distribution of the thick tail asymmetry. But the difficulty lies in the random process of choice, if the choice is undeserved, the calculated VaR will deviate from the actual value.

\subsection{The Steps of Calculate the Stock Portfolio by Copula-VaR Method}

Based on the above discussion and analysis, this paper will attempt to apply Copula-VaR method to the stock portfolio of calculation, and consider the stock portfolio has two kinds of price related commodity as the pledge; application specific steps are as follows:

Step 1, select two kinds of prices changes related products, and collect the historical price data of these two commodities.

Step 2, preliminary analysis price fluctuation of two types of goods, such as by price trend comparison to observe correlation between the two types of goods price fluctuations.

Step 3, two kinds of commodity returns whether obey the normal distribution or not, can examine each statistical characteristics, and do K-S test.

Step 4, choose appropriate Copula function, using the Kendall correlation coefficient and Spearman correlation coefficient to measure two kinds of commodity price changes and correlation, estimate relative parameters, and use P-value method for testing goodness of fit.

Step 5, use the Monte Carlo simulation method to calculate VaR based on Copula inventory combination function, and do precision test.

Step 6, calculate the mortgage rate of stock portfolio according to mortgage rate model.

\section{Computed Inventory Combination VaR}

\subsection{The Selection of Pledge}

This paper selects copper and zinc as a stock portfolio of mortgage financing. On the one hand, both in the spot market and futures market, each contract variety price is continuous, easy to collect and analysis. On the other hand, there is a certain correlation between the two kinds of non-ferrous metals market demand. Copper is widely used in automobile manufacturing, construction industry, machinery manufacturing, defense industry, light industry etc. Total consumption of zinc in approximately half of the proportion was used in galvanized industry, while zinc is also widely used in automobile manufacturing, ship building industry, light industry, etc. Zinc itself strength and hardness is not enough, so often join copper and other alloy elements, in order to improve the strength and hardness, which used in automotive, construction, electrical, mechanical manufacturing etc. The copper and zinc are also used in many industries, so when the demand for zinc is increased, the demand for copper will increase, leading to zinc prices rise, copper prices will rise; when the zinc demand reduction, the demand for copper will be reduced, resulting in zinc and copper prices will fall. Copper and zinc prices is not only affected by the political environment, market demand, industry policy, international market factors can influence of two kinds of product prices, therefore, the price trend will not be exactly the same, but has a strong correlation.

\subsection{Stock Portfolio Copula-VaR Calculation}

Through the Matlab software for Monte Carlo simulation of the pledge portfolio return rate, Fig. 1 is the simulation plot of 2000 times pledge yield marginal distribution points.

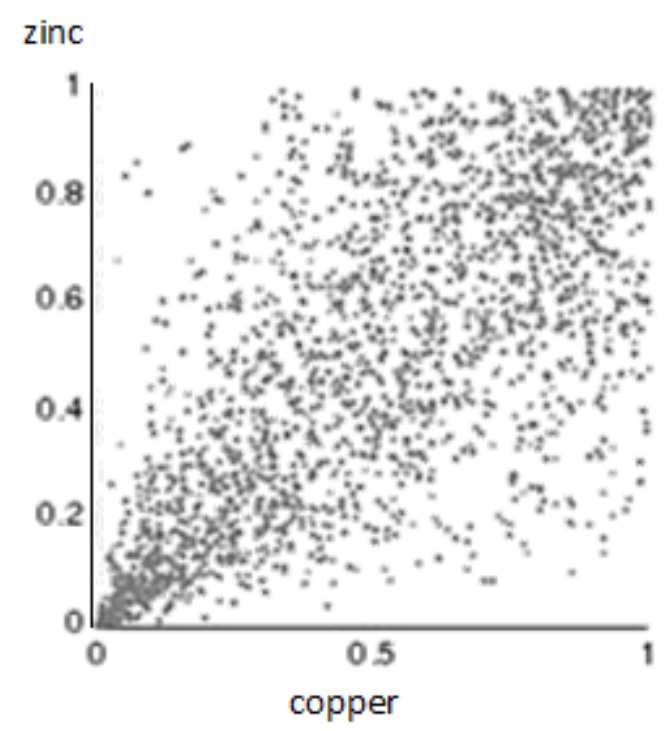

Figure 1. Scatter diagram of the pledge portfolio return rate

The $X$ axis of fig. 1 represents the 1\# copper simulation yield data; $Y$ axis represents $1 \#$ zinc yield data. From the scatter diagram, the lower tail part is more intensive, the upper tail part is more dispersed. It has asymmetric characteristics of upper and lower tail distribution; correspond with Clayton Copula distribution function, which further indicates the choice Clayton Copula has good fitting effect.

Based on the simulation data of 1000 times, 2000 times, and 5000 times, we can get a large number of experience distribution and pseudo random number, calculate the traditional VaR and Copula-VaR value at $95 \%$ confidence level. Calculation results of the traditional VaR and Copula-VaR are listed in "Tab. 1" below.

It is seen from Tab. 1, under different number of simulations times, Copula-VaR values are greater than the 
traditional VaR, using the Copula function can reflect more spike characteristics of the collateral prices, which can better estimate the loss when calculating the pledge value at risk.

Table 1. Calculation results of the traditional VaR and Copula-VaR

\begin{tabular}{llll}
\hline Different VaR & \multicolumn{3}{l}{ Simulation times } \\
\cline { 2 - 4 } value & $\mathbf{1 0 0 0}$ & $\mathbf{2 0 0 0}$ & $\mathbf{5 0 0 0}$ \\
\hline Traditional VaR & 0.0483 & 0.0489 & 0.0491 \\
Copula-VaR & 0.0599 & 0.0620 & 0.0653 \\
\hline
\end{tabular}

\subsection{VaR Calculation Accuracy Test}

Review test is used to test whether the VaR calculation method of model is accurate, also means that the VaR results to actual coverage in what extent of risk loss. For example, when calculate VaR at the $95 \%$ confidence level, the probability that the actual loss more than VaR should be less than $5 \%$, in this case, the VaR calculation results more accurate.

The most simple method to approve the accuracy of the model is record the failure rate, find out the given sample frequency exceeds VaR events. If banks provided the VaR value with $1 \%$ left end region $(p=1-c)$, within the total number of $\mathrm{T}$, the user counts the number of times the actual loss exceeds the previous day's VaR value. $\mathrm{N}$ is defined as the frequency of occurrence of failure events; N/T is the loss of efficiency. In the ideal case, loss of efficiency should be given no bias measure $p$, which should tend to $P$ with the increase of samples number. Kupiec give formulas of this method, the most suitable test is the likelihood ratio test to the null hypothesis $p=p^{*}$, statistic follows a chi-square distribution. Assuming the VaR model with time independent, failure days $N$ obeys Binomial distribution of $B(T, P)$, then,

$$
\begin{aligned}
L R_{u c}= & -2 \ln \left[(1-p)^{T-N} P^{N}\right] \\
& +2 \ln \left\{[1-N / T]^{T-N}(N / T)^{N}\right\}
\end{aligned}
$$

$L R_{u c}$ obeys the chi square distribution of one degrees of freedom, under the given confidence level, compare the actual test and theoretical test, we can judge the accuracy of VaR model. After getting the value of VaR, to test it in Matlab, there are 22 working days in a month, the test results are shown in "Tab. 2".

Table 2. The failure frequency test of traditional VaR and Copula-VaR

\begin{tabular}{llll}
\hline \multirow{2}{*}{ Failure frequency } & \multicolumn{4}{l}{ Simulation times } \\
\cline { 2 - 4 } & $\mathbf{1 0 0 0}$ & $\mathbf{2 0 0 0}$ & $\mathbf{5 0 0 0}$ \\
\hline Traditional VaR & $5.67 \%$ & $5.63 \%$ & $5.62 \%$ \\
Copula-VaR & $4.08 \%$ & $4.07 \%$ & $4.03 \%$ \\
\hline
\end{tabular}

It is seen from table 2, when the confidence level is $95 \%$, regardless of simulation times is 1000 times, 2000 times or 5000 times, Copula-VaR failure frequency is less than that of the traditional VaR. Copula-VaR can better measure the stock portfolio price risk. At the same time, table 2 also shows that when the confidence level is fixed, in the same way, the more simulation number, the less of failure frequency, the more effective of VaR value. The traditional VaR method calculate value at risk based on the assumption of asset return follows a normal distribution, ignoring the probability of extreme events, which do not well cover the business risks.

\subsection{The Pledge Rate of Stock Portfolio Calculation Based on Copula-VaR Model}

Calculation the pledge rate of initial stock portfolio by the traditional VaR method and Copula-VaR method, stock price take from the average annual price value, $t=0, p_{1,0}$ is average price of $1 \#$ copper, $q_{1,0}$ is $1 \#$ copper quantity, $p_{2,0}$ is average price for $1 \#$ zinc, $q_{2,0}$ is zinc number, take tons as the pledge number of units, and assume $q_{1,0}=q_{2,0}=1$, safety coefficient $\beta$ is 0.8 , calculates according to the above information and get $p_{1,0}=57699.74, p_{2,0}=15115.60$. At the $95 \%$ confidence level, calculates the pledge rate under different VaR method conditions for 1000 times, 2000 times, 5000 times, respectively, bring the above conditions into the pledge rate calculation formula $\theta=\frac{\sum p_{n, t} q_{n, t}-V a R_{t}}{\sum p_{n, t} q_{n, t}} \times \beta$, the results lists in "Tab. 3".

Table 3. Calculation results of the pledge rate under different method of $\operatorname{VaR}$

\begin{tabular}{llll}
\hline \multirow{2}{*}{ VaR Method } & \multicolumn{4}{l}{ Simulation times } \\
\cline { 2 - 4 } & $\mathbf{1 0 0 0}$ & $\mathbf{2 0 0 0}$ & $\mathbf{5 0 0 0}$ \\
\hline Traditional VaR & 0.76136 & 0.76088 & 0.76072 \\
Copula-VaR & 0.75208 & 0.75040 & 0.74776 \\
\hline
\end{tabular}

It can be seen from table 3 , mortgage rate calculated by using the Copula-VaR method is less than that of traditional VaR method, this is because the Copula function more fully considering the correlation between the fluctuations of the collateral price, and thus more cautious about the risk of inventory combined pledge business, reduce the risk of lending institutions.

\section{Conclusion}

When enterprise finance inventory, may not only one type of inventory, and there is a strong correlation between the stock price changes. So, as lenders in the mortgage rate, if we do not consider this correlation, view the two inventory as independent two pledge, set pledge rate respectively, then they will not avoid one pledge prices change causes another pledge price drop, which bring greater risk of inventory financing. Based on this idea, this paper makes an empirical calculation, according to the calculation results; the following conclusions can be drawn.

In practice, when lenders set the pledge rate, usually according to the empirical valuation, no verification system and scientific calculation method. The inventory 
combination pledge rate is computed by Copula-VaR method, which is a discussion of inventory financing and risk management. Specifically, is to explore the quantitative risk management of stock portfolio pledge business, to a certain inspiration and guidance to the practice of business. In the inventory pledge loan, one of the lenders face the risk is the cash risk of pledge, when borrowers unable to repay the loan, then the loan institution has the right to disposal pledge to compensate the risk. The pledge price movements has very important influence to pledge value, therefore, the pledge change cash is a major risk that lending institutions face. Especially when the pledge prices fall, the collateral value may be lower than the loan. When the stock price changes have strong correlation, and the inventory all used to pledge loans, loan institutions face greater cash risk. Therefore, it should take these stocks as a portfolio, to consider the portfolio risk.

In the value at risk calculation, the VaR method has two basic assumptions, one is the asset return follows a normal distribution, another is the return on assets is linearly related. But by calculating the statistical characteristics of stock returns and the test of normality was found, copper and zinc rate of return does not conform to normal distribution characteristics. The relationship between changes in the prices of stocks, not simply expressed in terms of the correlation coefficient. Because correlation coefficient can be expressed as linear relationship, cannot describe the nonlinear relationship, introducing the Copula function can capture the nonlinear relationship between assets. Value at risk calculations of risk inventory combination using Copula function than with VaR directly calculated, because the Copula function better describe the correlation between price changes of stock, can cover risks in price change. Copula-VaR method calculation result is lower than that of VaR method, for the lending institutions, which can inventory pledge business risk.

When calculate the inventory combination pledge rate, Copula-VaR applies to price changes have a strong correlation, with the two inventory portfolio for example, such as a stock prices fall lead to another stock prices fall, or a stock prices increase lead to another survival prices dropped. In using this method, should analyze dependence structure by using historical data, so the inventory price data should be easy obtained, such as inventories in the futures market or the stock market listed species.

\section{Acknowledgements}

The study is supported by Beijing Wuzi University scientific research base of science and technology innovation platform of modern logistics, information and control technology research (project code: PXM2014_014214_000086).

\section{References}

[1] Jokivuolle E, Peura S. "Incorporating collateral value uncertainty in loss given default estimates and loan-to-value ratios", European Financial Management, vol.9, pp.299-314, Mar. 2003.

[2] Cossin D, Hricko T., "Astructural analysis of credit risk with risky collateral: Amethodology for haircut determination", Economic Notes, vol.32, pp.243-282, Feb.2003.

[3] Li Yixue, Xu Yu, Feng Gengzhong. "Domestic and foreign researches on the evolution of inventory financing", Research on economics and management, No.3,pp.22-26, Mar.2007.

[4] Qi Ershi, Ma Shanshan, Han Tie, "Study on Loan-to-Value Ratios of Basket Warehouse Receipt Hypothecating in Logistics Finance", Journal of Xidian University(Social Science Edition), vol.16,No.6, pp.50-53, Nov.2008.

[5] Gao Jie, Guo Shanshan, Feng Shanshan, "Research on loan-to-value ratios in the mode of dynamic logistics supervision and control", Logistics engineering and management, vol31, pp.39-40, Oct.2009.

[6] Sun Chaoyuan, Wei Yan, "Research on Loan-to-Value Ratio of Double Category Inventory Fiancing", Finance \& Economics, vol.283, pp.117-124, Oct.2011. 